\title{
Os riscos do uso indiscriminado de Femproporex como inibidor de apetite: uma
}

\section{revisão}

\author{
The risks of indiscriminate use of Femproporex as an appetite inhibitor: a review \\ Los riesgos del uso indiscriminados Femproporex como inhibidor de apetito: uma revisión
}

Recebido: 30/09/2021 | Revisado: 09/10/2021 | Aceito: 13/10/2021 | Publicado: 16/10/2021

\author{
Gustavo Henrique Sebold \\ ORCID: https://orcid.org/0000-0002-7428-5016 \\ Centro Universitário da Fundação Assis Gurgacz, Brasil \\ E-mail: ghsebold@ minha.fag.edu.br \\ Vagner Fagnani Linartevichi \\ ORCID: https://orcid.org/0000-0002-2624-7744 \\ Centro Universitário da Fundação Assis Gurgacz, Brasil \\ E-mail: linartevichi@fag.edu.br
}

\begin{abstract}
Resumo
A obesidade é reconhecida pela Organização Mundial de Saúde (OMS) como sendo o excesso de gordura do corpo, que promove prejuízos à saúde e qualidade de vida dos indivíduos. Deste modo, emergem diversas formas e tratamentos para essa doença, que podem ou não incluir a ação medicamentosa, dentre um dos medicamentos utilizados para o tratamento dessa patologia, está o Femproporex, que é um inibidor de apetite, cuja atuação se dá no sistema nervoso central, causando a sensação de saciedade, contudo, explicita-se como um problema a utilização indiscriminada desse medicamento. Deste modo, pautado na metodologia de revisão descritiva-exploratória, com base em 10 diferentes autores cujos materiais foram disponibilizados nas plataformas Scielo e Pubmed, buscou-se evidenciar quais são os principais riscos da utilização descomedida desse fármaco, bem como a possível atuação do profissional de farmácia sob a ótica da atenção farmacêutica. Sendo assim, notou-se que o público que mais faz uso desse fármaco são mulheres e os efeitos colaterais mais relatados são dores de cabeça, náuseas, insônia, tremores, entre outros. Deste modo, pode-se evidenciar que o farmacêutico é um profissional extremamente importante, contribuindo para a disseminação de informações e de conscientização da sociedade, buscando reduzir o uso indiscriminado e consequentemente os danos advindos da utilização irrestrita.
\end{abstract}

Palavras-chave: Depressores do apetite; Obesidade; Assistência farmacêutica.

\begin{abstract}
Obesity is recognized by the World Health Organization (WHO) as excess body fat, which impairs the health and quality of life of individuals. There are several forms and treatments for this disease, which may or may not include drug action. One of the drugs used to treat this disease is Femproporex, which is an appetite suppressant that acts on the central nervous system, causing a feeling of satiety, however, the indiscriminate use of this drug can be understood as a problem. Thus, based on the descriptive-exploratory review methodology, based on 10 different authors whose materials were made available on the Scielo and Pubmed platforms, we sought to highlight the main risks of the excessive use of this drug, as well as the possible role of the professional of pharmacy from the perspective of pharmaceutical care. It was found that the public that most uses this medication are women and the most reported side effects are headache, nausea, insomnia, tremors, among others. Thus, it can be seen that the pharmacist is an extremely important professional, contributing to the dissemination of information and awareness in society, seeking to reduce indiscriminate use and, consequently, the damages resulting from unrestricted use.
\end{abstract}

Keywords: Appetite depressants; Obesity; Pharmaceutical care.

\section{Resumen}

La obesidad es reconocida por la Organización Mundial de la Salud (OMS) como siendo el exceso de grasa en el cuerpo, que causan problemas a la salud y a la calidad de vida de los individuos. De este modo emergen diversas formas y tratamientos para esa enfermedad, que pueden o no incluir la acción medicamentosa, de entre uno de los medicamentos utilizados para el tratamiento de esa patología, está el Femproporex, que es un inhibidor de apetito, cuya actuación se da en el sistema nervioso central (SNC), causando la sensación saciedad, pero, se explicita un problema la utilización indiscriminada de esta medicina. De este modo, guiado en la metodología de revisión descriptiva-exploratoria, con base en 10 diferentes cuyos materiales fueron disponibles en las plataformas Scielo y Pubmed, se buscó evidenciar cuales son los principales riesgos de la utilización descomedida de este fármaco, tal como la posible actuación del profesional de farmacia bajo la óptica de atención farmacéutica. Así que se notó que en el público que más han utilizado este fármaco han sido las mujeres y los efectos colaterales más relatados son dolores 
de cabeza, náuseas, insomnio, temblores, entre otros. De este modo se puede evidenciar que el farmacéutico es un profesional extremamente importante, contribuyendo para la diseminación de informaciones o de concientización de la sociedad, buscando reducir el uso indiscriminado y consecuentemente los daños advenidos de la utilización irrestricta.

Palabras clave: Depresores del apetito; Obesidad; Cuidados farmacéuticos.

\section{Introdução}

Compreendida como uma doença multifatorial, pode-se considerar que a obesidade é uma das patologias que mais afeta pessoas no mundo, sendo também uma temática que vêm desvelando inúmeras pesquisas e contribuições perante a comunidade científica, sobretudo na área da saúde (Maria \& Yaegashi, 2016). Por vezes, a obesidade pode ser silenciosa, não possibilitando ao indivíduo que está sendo acometido pela patologia perceber os sintomas e buscar ajuda profissional. Alguns dos fatores que mais destacam-se em relação a essa doença englobam questões genéticas, emocionais, ambientais, psicossociais (Wanderley \& Ferreira, 2010). A Organização Mundial de Saúde (OMS) considera a doença como uma grande questão de saúde pública, comparando-a a uma pandemia, uma vez que pode vir a acometer todos os indivíduos, independentemente de faixa etária, classe social, trabalho ou nível escolar, tendo fatores predisponentes ou não.

Deste modo, além de demandas fisiopatológicas da obesidade, nota-se que os indivíduos constantemente buscam a aceitação corporal, atenuada pela ascensão das mídias e redes sociais, que o tempo todo exploram corpos padronizados. Neste sentido, emerge uma necessidade da busca pelo "corpo perfeito" e, para isso, a utilização medicamentosa de anorexígenos também demonstra ser uma possibilidade para alcançar o tão sonhado e desejado corpo perfeito (Lordani, 2019).

Nesta perspectiva, dada a demanda por medicamentos que visam a redução drástica do peso corporal, dietas, exercícios e procedimentos estéticos com o mesmo intuito, destaca-se a atuação do fármaco Femproporex, que tal qual os outros anorexígenos, tem uma atuação direta sobre a atuação neurotransmissora serotoninérgica e também catecolaminérgica, atuando como um inibidor de apetite, causando a sensação de saciedade, devido a atividade neurotransmissora noradrenérgica, responsável pela inibição do apetite (Duarte et al., 2020).

No Brasil há uma legislação específica que discorre acerca da utilização deste medicamento, cujo controle rigoroso faz parte de uma de suas demandas, sendo comercializado apenas com prescrição também retenção de receita médica. No ano de 2011, em território nacional, a Agência Nacional de Vigilância Sanitária (ANVISA) vetou a comercialização do Femproporex sob a alegação de que esse medicamento tinha potencial causador de patologias cardíacas em pacientes que faziam seu uso. Contudo, em 2014, pautado em estudos realizados pela European Medicines Agency (EMA) a ANVISA passa a liberar novamente a produção, comercialização e uso desse fármaco, juntamente com outros da mesma classe (Duarte et al., 2020). Estudos apontam que ainda são existentes resquícios do uso indiscriminado desse medicamento, sendo que a proibição não faria com que os indivíduos deixassem de utilizá-lo, sendo que sua retirada de circulação do mercado farmacêutico apenas acentuaria a utilização irrestrita do fármaco, apenas atenuando seu potencial negativo (Silva; Rodrigues; Bonelli, 2019).

Destarte, a atenção farmacêutica tem um papel de extrema relevância em relação à aquisição medicamentosa e a utilização correta dos fármacos destinados ao tratamento da obesidade. Além disso, essa assistência atua como uma forma de conscientizar os indivíduos e disseminar informações de cunho científico, a fim de que sejam evitados danos aos pacientes bem como o auxílio correto para o tratamento eficaz (Silva; Santana; Martins, 2021).

\section{Metodologia}

Para a realização deste estudo, escolheu-se a metodologia de revisão descritiva-exploratória de literatura (Estrela, 2018), cujo intuito foi apresentar considerações já elaboradas, a fim de que os resultados sejam atualizados, pautando-se em estudos mais antigos, a fim de que novas considerações sejam viabilizadas. 
Deste modo, foram realizadas 3 etapas para a elaboração do material, baseada na pergunta norteadora: Quais são os riscos da utilização do Femproporex enquanto um anorexígeno inibidor de apetite? Primeiramente, realizou-se uma busca geral nas plataformas de pesquisa acadêmica Scielo e Pubmed, com base nos seguintes Descritores em Ciências da Saúde (DeCS): Femproporex, anorexígeno, inibidor de apetite, obesidade e atenção farmacêutica.

Após, aplicaram-se critérios mais específicos para a seleção da amostragem, baseados no período correspondente a 2008-2021, cuja escrita encontrava-se em língua portuguesa e variações em língua inglesa. Foram incluídos materiais que contemplavam em sua metodologia revisões sistemáticas, estudos de caso, estudos descritivos transversais e pesquisas exploratórias (dados organizados segundo Minella \& Linartevichi, 2021).

Inicialmente, encontrou-se um total de 52 artigos que continham no mínimo 2 descritores supracitados. Em uma análise precisa a partir da leitura dos resumos foram descartados os trabalhos que não contemplavam o objetivo, que não expunham resultados diante da utilização indiscriminada do femproporex e outros fármacos anorexígenos. Seguindo uma análise ainda mais minuciosa, consistindo na leitura integral e fichamento do material, foram selecionados 10 artigos que estavam dentro dos critérios da revisão descritivo-exploratória e que atendiam aos objetivos e à justificativa proposta pelo estudo.

\section{Resultados}

As diversas compreensões sobre a obesidade, apontam, de alguma forma, para a necessidade de um tratamento que inclua tanto a administração de medicamentos quanto a transformação de hábitos e práticas que atrelam e/ou substituem a necessidade do uso de medicamentos. A Associação Brasileira para o Estudo da Obesidade (ABESO) aponta para um problema epidêmico de saúde pública, tanto a nível brasileiro quanto a nível mundial, sendo indicado através dos níveis de Índice de Massa Corporal (IMC).

Na Tabela 1, evidencia-se as principais considerações entre o material selecionado, destacando principalmente o uso irrestrito de medicamentos para emagrecimento. O principal foco é o Femproporex, contudo, outros fármacos também são descritos pelos pesquisadores.

Tabela 1. Comparativo entre os achados de literatura especializada.

\begin{tabular}{|c|c|c|c|}
\hline Fonte & & Título & Resultados \\
\hline $\begin{array}{l}\text { Lucas, } \\
\text { Teixeira } \\
\text { Oliveira } \\
(2021)\end{array}$ & $\&$ & $\begin{array}{l}\text { Farmacoterapia da obesidade: } \\
\text { uma revisão de literatura. }\end{array}$ & $\begin{array}{l}\text { Evidenciaram como efeitos não desejados do Femproporex a insônia, } \\
\text { alucinações, tremores, confusão mental, depressão, cefaleia, disforia, fadiga e } \\
\text { náuseas, sendo esse medicamento contraindicado para pacientes que já } \\
\text { possuem um histórico de doenças cardiovasculares, psiquiátricas ou glaucoma. } \\
\text { Expressam através do estudo a necessidade de conscientização do paciente, } \\
\text { uma vez que o tratamento com o Femproporex inclui contraindicações que } \\
\text { devem ser levadas a sério. Ressaltam que o fármaco possui um potencial de } \\
\text { redução da obesidade bastante considerável, contudo, deve estar atrelado à um } \\
\text { estilo de vida saudável, proporcionado por transformações que não são } \\
\text { advindas apenas do tratamento farmacológico. }\end{array}$ \\
\hline
\end{tabular}

Silva, Santana Uso dos anorexígenos, seus \& Martins riscos e farmacologia para o (2021)
Os autores apontam para a necessidade da assistência farmacêutica visando o uso racional do Femproporex, pois a falta de informação é um fator desvantajoso ao uso correto, bem como ao tratamento eficaz da obesidade. Antes de buscar a interação medicamentosa, é essencial que os indivíduos procurem orientação profissional multidisciplinar, englobando práticas físicas e apoio nutricional e médico. $\mathrm{Na}$ percepção dos autores a utilização de medicamentos para a finalidade de emagrecimento deve acontecer apenas em últimos casos, visando opções alternativas e menos danosas ao organismo dos indivíduos, sendo que quando o tratamento farmacológico é inevitável, deve-se atentar para a prescrição de fármacos cujos efeitos colaterais sejam mínimos e toleráveis e que o prazo de utilização desses medicamentos não ultrapasse a 
Duarte et al., Uso de anfepramona, (2020)

femproporex, mazindol e sibutramina no tratamento de pacientes com sobrepeso ou obesidade: análise farmacológica e clínica.

Oliveira \& Riscos do uso indiscriminado Fattori (2020) de anorexígenos para o tratamento de sobrepeso.
Silva, Bonelli (2019)
Rodrigues \&

$\mathrm{O}$ risco do uso dos anorexígenos disponíveis no Brasil para o tratamento da obesidade.

Radaelli,

Pedroso

Medeiros

(2016)

Moreira \&

Alves (2015)

Utilização de anfetaminas como anorexígenos relacionadas à obesidade.

Mota et al., (2014)

Rocha,

Fernandes \&

Garcia (2012)

Avaliação do consumo de anorexígenos derivados de Anfetamina em cidades de margem dos 30 dias, evitando a dependência química.

Os autores verificam que o Femproporex tem alto potencial de emagrecimento, contudo, na atualidade, percebe-se o uso irracional desse e de outros medicamentos com o mesmo intuito. Salienta-se efeitos graves decorrentes do uso desmedido do medicamento, tal como hipertensão arterial, mudanças bruscas de humor, taquicardia, insônia, além de dependência química e física.

Asseverou-se que os riscos da utilização desmedida de anorexígenos como o Femproporex pode causar problemas relacionados à saúde física e também psicológica dos usuários, tendo como exemplo: taquicardia, hipertensão arterial, insônia, irritabilidade, ansiedade, depressão e dependência química, variando de acordo com o organismo de cada indivíduo. Os autores reforçam que a obesidade se trata de um problema de saúde pública que pode ser agravado com o uso irresponsável de anorexígenos. Para a efetividade destes, é primordial o acompanhamento de um médico e a mudança relacionada à qualidade de vida, como a reeducação alimentar, a prática de exercícios, o acompanhamento nutricional, entre outros aspectos.

As autoras ressalvaram a importância de realizar maiores pesquisar comprovando a eficácia e segurança do Femproporex e de outros fármacos utilizados para a inibição de apetite, uma vez que até 2019 a quantidade de riscos à utilização de anorexígenos ainda era superior aos benefícios proporcionados por eles. Ademais, relatou-se a importância de realizar um trabalho voltado à conscientização dos usuários, levando informações científicas e trabalhando com a reeducação nutricional, de modo a evitar a utilização de fármacos para o tratamento da obesidade.

Os autores indicam que, estatisticamente, no ano de 2004 o Brasil possuía $100 \%$ da produção mundial de Femproporex, importando cerca de 99,6\%. Em relação ao tratamento com drogas anorexígenas, sobretudo o Femproporex, percebe-se a necessidade de atrelar o tratamento medicamentoso a outras práticas, tais como exercícios físicos, reeducação alimentar, entre outros, a fim de que diante da interrupção do tratamento, o paciente não venha a ganhar o peso que perdeu durante a interação do medicamento. Defende a atuação do farmacêutico enquanto um orientador, sendo parte do tratamento seguro e eficaz poder conscientizar os pacientes e acompanhá-los, visando expor todos os riscos decorrentes da utilização do remédio, além de não oportunizarem acesso facilitado ao medicamento para pacientes que não possuem prescrição médica.

Os autores evidenciam que um dos fármacos mais procurados com finalidade de emagrecimento é o Femproporex, dentre as principais reações desse fármaco, destacam-se: hipertensão arterial e pulmonar, glaucoma, náuseas, vômitos, cefaleia, ansiedade e palidez. Os autores verificam que quando esse medicamento interage com o álcool e outras substâncias, pode causar efeitos colaterais ainda mais potencializados e até mesmo dependência química. Ressalta-se a falta de informação como um fator intensificador ao uso irresponsável deste e de outros medicamentos, os autores defendem a conscientização da sociedade para a utilização responsiva do Femproporex e outras drogas.

Os autores verificam que a quantidade de consumo dos inibidores de apetite não acompanha a distribuição populacional diagnosticada com obesidade, revelando um consumo desenfreado desses fármacos, atentando para uma possível problemática no uso racional desses medicamentos. Demonstrou-se a partir da pesquisa que mesmo em um período cuja comercialização do Femproporex era proibida no país, o medicamento ainda estava presente nos dados, sendo a proibição uma contribuição apenas em partes para a redução do uso irracional do fármaco. Goiás.
O Femproporex foi considerado o $3^{\circ}$ fármaco mais utilizado entre os participantes da pesquisa, correspondendo a um percentual de $21 \%$ dos participantes. Destes $21 \%$, A grande maioria dos participantes relatou sentir algum efeito adverso ao utilizar o fármaco, tais como insônia, dores de cabeça, ansiedade, tremores e palpitações e tonturas. Cerca de 50,5\% dos voluntários afirmam utilizar o medicamento apenas com o intuito de perder peso, podendo estar atrelado ou não com exercícios físicos e a reeducação alimentar. Cerca de 45,8\% dos participantes buscaram auxílio médico para fazer uso do 
Toledo \& Uso de medicamentos para Colaboradores perda de peso e índice de massa (2010) corporal em universitários do Vale do Araguaia (MT/GO), Amazônia Legal. medicamento, 31,7\% foram influenciados por pessoas que já faziam uso e $23,5 \%$ obtiveram o medicamento em farmácias sem a receita médica, apenas por interesse próprio. $27,5 \%$ dos participantes relataram ter tido orientação médica e $31,7 \%$ afirmaram ter tido assistência farmacêutica para o uso do fármaco.

O estudo evidenciou que cerca de $9 \%$ dos alunos entrevistados faziam o uso de algum medicamento voltado especificamente para o emagrecimento. Percebeuse que um dos fármacos mais utilizados e populares entre a comunidade acadêmica era o Femproporex (6,7\%). A maior parte dos adeptos ao medicamento eram mulheres com idade superior a 30 anos, com sobrepeso. Apenas $41 \%$ dos estudantes evidenciaram ter recebido informações médicas para a utilização do medicamento.

Fonte: Autores (2021).

Apontam-se evidências da utilização do Femproporex para a perda de peso desde o início da década de 70, embora ainda se perceba a carência acerca de estudos sobre a temática e seus efeitos efetivamente positivos. Existem comparações deste fármaco com a Dextroanfetamina, postulando-se que o Femproporex deriva deste último, buscando a transformação da intencionalidade, visto que, enquanto o medicamento supracitado busca obter efeito anorexígeno, atuando no Sistema Nervoso Central, a Dextroanfetamina pressupõe efeitos psicoestimulantes, sobretudo para o tratamento de transtorno de déficit de atenção, por exemplo (Silva, Santana \& Martins, 2021; Lucas, Teixeira \& Oliveira, 2021; Duarte et al., 2020). O Femproporex atua diretamente sobre os centros hipotalâmicos, o que causa a inibição do apetite, sendo também uma segunda opção aos pacientes que não responderam positivamente ao tratamento com o fármaco Sibutramina (Cardozo et al., 2021; Duarte et al., 2020). A seguir, observa-se a composição química deste fármaco, conforme apontado por Franck (2008).

Destarte, o uso do Femproporex pode ser aconselhado à pacientes que possuam obesidade, cujo IMC ultrapasse $30 \mathrm{~kg} / \mathrm{m}^{2}$ ou então $25 \mathrm{~kg} / \mathrm{m}^{2}$ especificamente a pacientes que apresentem comorbidades inerentes à patologia supracitada (Lucas; Teixeira; Oliveira, 2021; Duarte et al., 2020; Moreira \& Alves, 2015).

Figura 1. Composição química do Femproporex.

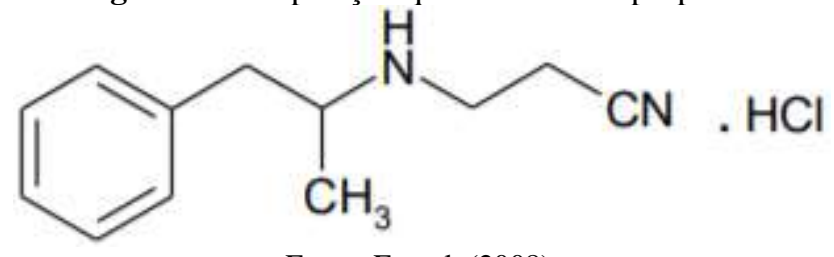

Fonte: Franck (2008),

Os artigos selecionados para a composição dessa revisão, englobam diferentes metodologias, contudo, apresentam contribuições que demonstram relativas semelhanças, principalmente no que tange à busca pelo tratamento farmacológico, incluindo a utilização do Femproporex, seja para o tratamento da obesidade ou não, e também a necessidade de orientação médica e farmacêutica para prescrição, acompanhamento e conhecimento da administração medicamentosa, bem como seus efeitos, conforme percebe-se através do comparativo estabelecido na tabela 1.

Os estudos apontaram que os efeitos colaterais mais sentidos pelos pacientes que fazem uso do Femproporex são: insônia, alterações no humor, taquicardia e boca seca. Como efeitos menos comuns, mas ainda existentes, agravados sobretudo pelo uso irracional do medicamento, em doses superiores ao recomendado pelo profissional médico, acentua-se a dependência química (Marcon et al., 2012; Silva; Oliveira \& Ferreira, 2012; Oliveira \& Fattori, 2020). A longo prazo, também são evidenciados a presença de efeitos negativos como hipertensão arterial, arritmia cardíaca, além do famoso efeito rebote - que se trata do retorno à condição inicial a que o paciente indicava estar, no início do tratamento (Oliveira \& Fattori, 2020; Silva; Rodrigues \& Bonelli, 2019). A tabela 2, a seguir demonstra os principais efeitos do uso do Femproporex, em conformidade à forma de administração. 
Tabela 2. Efeitos adversos do uso de Femproporex adaptado de Marcon e Colaboradores (2012).

\begin{tabular}{ll}
\hline Forma de uso & Reação adversa \\
\hline Doses baixas & $\begin{array}{l}\text { Inibição do apetite, náuseas, vômito, dores de cabeça, insônia, tremores, } \\
\text { taquicardia, hipertensão, movimento involuntário dos olhos, alucinações, boca } \\
\text { seca. }\end{array}$ \\
Doses elevadas & $\begin{array}{l}\text { Hipertensão, taquicardia, disritmia, alucinações visuais, crises de pânico, } \\
\text { irritabilidade, boca seca, constipação, parestesia, dependência química e óbito. }\end{array}$ \\
Efeitos residuais & Insônia, dores musculares e fadiga. \\
Efeitos crônicos & $\begin{array}{l}\text { Depressão, crises de pânico e ansiedade, psicose, perda acentuada de peso, } \\
\text { irritabilidade, fadiga, insônia. }\end{array}$ \\
\hline
\end{tabular}

Fonte: Adaptado de Marcon et al. (2012).

\section{Discussão}

Nota-se uma exacerbada cultura de utilização medicamentosa sem prescrição médica, inerente inclusive ao emprego de anorexígenos - incluindo o Femproporex - como uma forma fácil e rápida de perder peso, sobretudo a usuários cujo IMC é normal ou que apresentam sobrepeso (Mota et al., 2014; Silva; Santana \& Martins, 2021).

Demonstrou-se, a partir dos estudos que os indivíduos que buscam o auxílio do Femproporex com a finalidade de perder peso, constituem majoritariamente um público feminino, que já apresenta sobrepeso. Os autores alertam, porém, que o tratamento farmacológico, nesses casos, nem sempre se trata da solução mais eficaz, podendo ainda, trazer consequências extremamente prejudiciais para os pacientes (Toledo et al., 2010; Oliveira \& Fattori, 2020). A irresponsabilidade na administração do medicamento doravante a indiscriminalização da utilização de determinados fármacos e o senso comum diante das compreensões acerca do Femproporex e outros anorexígenos também alcançam indivíduos cujo IMC tem proporções normais, exacerbando diversos problemas de saúde aos pacientes que buscam realizar o tratamento medic amentoso sem prescrição e sem necessidade - por conta de distúrbios alimentares ou até mesmo pela vaidade acentuada pelo culto ao “corpo perfeito" (Silva; Rodrigues \& Bonelli, 2019; Santana; Silva \& Martins, 2021).

Os autores são unânimes em expor a necessidade de busca por orientação médica, uma vez que Femproporex se trata de um fármaco anorexígeno que apenas pode ser obtido com receitas do tipo B2, ficando retida pelo estabelecimento farmacêutico, devendo o tratamento ser igual ou inferior a 30 dias (Gusmão et al., 2021; Silva; Rodrigues \& Bonelli, 2019). Neste sentido, alerta-se que a dificuldade em comprar e fazer o uso desses medicamentos, derivam das atitudes irresponsáveis e da comercialização desregrada, que pode ser danosa para pacientes que não conhecem todos os efeitos, utilizam-se de dosagens superiores às recomendadas - no intuito de potencializar o efeito farmacológico - e ainda, colocam em risco a segurança de sua saúde (Oliveira \& Fattori, 2020; Silva; Rodrigues \& Bonelli, 2019; Mota et al., 2014). Deste modo, considera-se também que um dos maiores desafios da indústria farmacêutica é a elaboração de fármacos que sejam mais seguros aos pacientes, para tal finalidade, culminando em intervenções que afetem minimamente a qualidade de vida dos pacientes e cujos efeitos colaterais sejam toleráveis (Mota et al, 2014; Radaelli; Pedroso \& Medeiros, 2016; Duarte et al., 2020).

Apesar de em momentos históricos específicos a comercialização do Femproporex tenha sido proibida, ainda se nota que isso não impediu que os usuários - com obesidade ou não - consumissem esse fármaco, e que inclusive, o Brasil fosse um dos maiores exportadores desse medicamento para outros países, estando ele proibido ou não (Radaelli; Pedroso \& Medeiros, 2016). Isso demonstra que o uso responsivo e consciente deste - e outros medicamentos relacionados - é muito mais eficaz do que a própria proibição (Radaelli; Pedroso \& Medeiros, 2016; Duarte et al., 2020; Silva; Rodrigues \& Bonelli, 2019).

É sabido que, por vezes, a dificuldade em encontrar a orientação médica acaba levando os indivíduos a buscarem alternativas mais rápidas e facilitadas, fazendo com que, infelizmente, muitos pacientes acabem aderindo a tratamentos que 
põem em risco sua saúde, bem como demonstram ineficácia, sem qualquer comprovação advinda da comunidade científica. Outrossim, a assistência farmacêutica deve ser considerada e trabalhada, no sentido de auxiliar os pacientes, haja posto a contribuição social que estes podem oferecer para a sociedade, diminuindo inclusive impactos do uso indiscriminado do Femproporex e outros fármacos que necessitam de uma maior atenção (Moysés et al., 2021; Silva; Santana \& Martins, 2021; Mota et al., 2014).

Nesta perspectiva a figura do farmacêutico é peça chave no auxílio, através da assistência farmacêutica, para a compreensão da utilização de anorexígenos, bem como a administração correta, evitando seu mal uso. Admite-se que o farmacêutico tem um papel primordial de auxílio aos pacientes, a partir das práticas de assistência farmacêutica, propondo também um rígido controle sobre a dispensação de medicamentos (Sousa et al., 2019).

O profissional farmacêutico também é importante no esclarecimento de dúvidas e na disseminação de informações científicas, de modo acessível a todos os pacientes, evidenciando as prováveis interações do medicamento, os efeitos colaterais, as diferentes possibilidades de tratamento que não envolvem o fármaco diretamente, o diagnóstico a partir de um médico competente sobre cada especialidade, além de consequentemente poder auxiliar nas questões de uso correto do medicamento, evitando a superdosagem, entre outros, que estabelecem-se enquanto um trabalho multidisciplinar (Radaelli; Pedroso \& Medeiros, 2016; Silva; Rodrigues \& Bonelli, 2019).

\section{Conclusão}

As pesquisas sobre os anorexígenos, principalmente o Femproporex, apontam para a necessidade de estudos que comprovem ainda mais sua eficácia no combate e tratamento à obesidade, esclarecendo aos usuários todos os aspectos positivos e negativos de seu uso. Nota-se que ainda há um relativo desconhecimento da população e de alguns usuários que praticam o uso indiscriminado desse fármaco, o que leva os pacientes a não pensarem e não ressignificarem suas práticas, levando a automedicação a ser um problema de saúde pública, sobretudo relacionada à medicamentos que devem ser devidamente controlados, por conta de seus efeitos adversos e prospectivos danos.

Como uso indiscriminado de Femproporex subentende-se a comercialização desmedida e descomplicada de um medicamento que já vêm demonstrando - através de estudos - que precisa de acompanhamento e orientação profissional para sua prescrição e administração, bem como a superdosagem com propostas de potencializar o efeito do fármaco, além do autodiagnóstico seguido da automedicação. Tudo isso reflete uma carência de políticas voltadas à atenção farmacêutica, enquanto uma possibilidade para a explanação de percepções acerca de riscos, culminando na conscientização da população em geral, estimulando a busca por tratamentos não farmacológicos, ou quando farmacológicos, com a devida orientação médica.

Por fim, é de grande valia que os profissionais e estudantes das áreas da saúde, seja a farmácia, medicina, enfermagem, entre outros, continuem pesquisando acerca da utilização do Femproporex e de outros anorexígenos, a fim de comprovar a eficácia do medicamente de forma segura - quando houver - e disseminar informações para a população em geral, fazendo com que a utilização irresponsável seja freada e que os indivíduos consigam compreender quais são os riscos a que estão submetidos quando permitem-se utilizar um fármaco sem a mediação, prescrição e acompanhamento de um profissional da área.

Deste modo, sugere-se que novos estudos devam ser realizados, principalmente aqueles que evidenciam a necessidade de que políticas de atenção farmacêutica juntamente com trabalhos multidisciplinares a equipes nutricionais e médicas sejam propostos, como uma forma de sensibilizar e orientar a sociedade sobre utilização de Femproporex sem a devida prescrição, como uma maneira de minimizar os riscos do uso indiscriminado, que podem afetar negativamente a vida desses usuários. 


\section{Referências}

Cardozo, A. G. L., Rosa, R. L., Novak, R. S., Folquitto, D. G., Schebelski, D. J., Brusamarello, L. C. C., \& Ribeiro, D. T. B. (2021). Erva-mate (Ilex paraguariensis A. St. - hil.): uma revisão abrangente sobre composição química, benefícios à saúde e recentes avanços. Research, Society and Development, 10(11), e590101120036. https://doi.org/10.33448/rsd-v10i11.20036

De Maria, C. C. \& Yaegashi, S. F. R. (2016). Os traços de personalidade associados no desenvolvimento da obesidade Revista Brasileira de Obesidade, Nutrição e Emagrecimento, 10(56), 74-92. http://www.rbone.com.br/index.php/rbone/article/view/411

Duarte, A. P. C., Govato, T. C. P., Carvalho, R. G., Pontes-Junior, L. C. B., Rodrigues, C. L., Santos, G. M. P., Nicolau, L. A. D., Ferraz, R. R. N. \& Menezes-Rodrigues, F. S. (2020). Uso de Anfepramona, Femproporex, Mazindol e Sibutramina no tratamento de pacientes com sobrepeso ou obesidade: análise farmacológica e clínica. International Journal of Health Management Review, 6(2), 1-8. https://ijhmreview.org/ijhmreview/article/view/210/142

Estrela, C. (2018). Metodologia Científica: Ciência, Ensino, Pesquisa. Editora Artes Médicas.

Franck, M. C. (2008). Desenvolvimento e validação de metodologia analítica para caracterização e quantificação de derivados anfetamínicos. Dissertação de Mestrado. UFRGS.

Gusmão, A. B. de., Rocha, S. de S., Ferreira, B. W. R. C., Santos, A. K. F. de S., \& Macedo, C. L. (2021). Manejo nutricional e farmacológico da obesidade pediátrica: um tratamento multiprofissional. Research, Society and Development, 10(1), e60010111797. https://doi.org/10.33448/rsd-v10i1.11797

Lordani, C. R. F. (2019). Representações sociais de sujeitos em situação de obesidade considerada grave: trajetórias de vida e itinerários terapêuticos no Sistema Único de Saúde (SUS). Tese de Doutorado. UNISINOS-SP. São Leopoldo.

Lucas, B. B., Teixeira, A. P. C. \& Oliveira, F. S. (2021) Farmacoterapia da obesidade: uma revisão de literatura. Journal of Biology \& Pharmacy and Agricultural Management, 17(1), 165-185. http://revista.uepb.edu.br/index.php/biofarm/article/view/5723/3478

Marcon, C., Silva, L. A. M., Moraes, C. M. B., Martins, J. S. \& Carpes, A. D. (2012). Uso de anfetaminas e substâncias relacionadas na sociedade contemporânea. Disciplinarum Scientia, 13(2), 247-263. https://periodicos.ufn.edu.br/index.php/disciplinarumS/article/viewFile/1018/963.

Minella, F. C. O., \& Linartevichi, V. F. (2021). Efeitos do canabidiol nos sinais e comorbidades do transtorno do espectro autista. Research, Society and Development, 10(10), e64101018607. https://doi.org/10.33448/rsd-v10i10.18607

Moreira, F. \& Alves, A. A. Utilização de anfetaminas como anorexígenos relacionados à obesidade. Revista Científica da FHO, 3(1), 84-91. http://www.uniararas.br/revistacientifica/_documentos/art.9-029-2015.pdf

Mota, D. M., Oliveira, M. G., Bovi, R. F., Silva, S. F., Cunha, J. A. F. \& Divino, J. A. (2014) Há irracionalidades no consumo de inibidores de apetite no Brasil? Uma análise farmacoeconométrica de dados em painel. Ciência \& Saúde Coletiva, 19(5), 389-400. http://orcid.org/0000-0003-4156-0138

Moysés, D. de A., Antunes, A. R. F., Santos, D. de N. A. dos., Correa, R. M. dos S., Galucio, N. C. da R., Gonçalves, T. V., Teixeira, E. B., Silva, M. G. de O. P. da, Rocha, A. A., Costa, J. G. da., Medeiros, E. R. do N., Silva, W. da C., \& Vale, V. V. (2021). Efeitos colaterais relacionados a suplementos termogênicos que contém sinefrina . Research, Society and Development, 10(10), e269101018828. https://doi.org/10.33448/rsd-v10i10.18828

Oliveira, E. R., \& Fattori, N. C. M. (2020). Riscos do uso indiscriminado de anorexígenos para o tratamento de sobrepeso. Revista Científica Eletrônica de Ciências Aplicadas da FAIT, 1(2), 1-14.

Radaelli, M., Pedroso, R. C., \& Medeiros, L. F. (2016). Farmacoterapia da obesidade: benefícios e riscos. Revista Saúde e Desenvolvimento Humano, 4(1), 101-115. http://dx.doi.org/10.18316/2317-8582.16.23

Silva, F. I. L., Rodrigues, G., \& Bonelli, O. A. (2019). O risco do uso dos anorexígenos disponíveis no Brasil para o tratamento da obesidade. Revista Saberes da Faculdade de São Paulo, 9(1), 1-10.

Silva, J. R., Oliveira, E. N. F., \& Ferreira, A. G. (2012). Avaliação do consumo de anorexígenos derivados de anfetamina em cidades de Goiás. Ensaios $e$ Ciência: Ciências Biológicas, Agrárias e da Saúde, 16(3), 9-19. https://www.redalyc.org/pdf/260/26029237001.pdf

Silva, R. C. N., Santana, C. A., \& Martins, T. S. (2021). Uso dos anorexígenos, seus riscos e farmacologia para o tratamento da obesidade. Revista Científica Multidisciplinar Núcleo do Conhecimento, 6(4), 124-140.

Sousa, D. M. D. de, Sousa, M. D. de, Macedo, J. L., Silva, S. S. da, Silva, R. R. C. da, Brito Nascimento, L. L., Santos, L. S. dos, \& Miranda Junior, R. N. C. (2019). Fitoterápicos utilizados para perda de peso comercializados em farmácias. Research, Society and Development, 8(4), e184930. https://doi.org/10.33448/rsd-v8i4.930

Toledo, O. R., Castro, J. A. M., França, A. C. H., \& Ferrari, C. K. B. (2010). Uso de medicamentos para perda de peso e índice de massa corporal em universitários do Vale do Araguaia (MT/GO), Amazônia Legal. Revista Brasileira de Clínica Médica, 8(6), 480-486. http://files.bvs.br/upload/S/16791010/2010/v8n6/a1595.pdf

Wanderlei, E. N., \& Ferreira, V. A. (2010). Obesidade: uma perspectiva plural. Revista Ciência e Saúde Coletiva, 15(1), 185-194. https://doi.org/10.1590/S1413-81232010000100024 